\title{
Marital Status and Cognitive Impairment among Community-Dwelling Chinese Older Adults: The Role of Gender and Social Engagement
}

\author{
Lei Feng ${ }^{a} \quad$ Xue-Ting $\mathrm{Ng}^{\mathrm{b}}$ Philip Yap ${ }^{\mathrm{c}}$ Jialiang Li ${ }^{\mathrm{b}} \quad$ Tih-Shih Lee $^{\mathrm{d}}$ \\ Krister Håkansson ${ }^{e}$ Ee-Heok Kua ${ }^{a}$ Tze-Pin $\mathrm{Ng}^{\text {a }}$ \\ a Department of Psychological Medicine, Yong Loo Lin School of Medicine, and \\ ${ }^{b}$ Department of Statistics and Applied Probability, National University of Singapore, \\ 'Department of Geriatric Medicine, Khoo Teck Puat Hospital, and d Neurobehavioral \\ Disorders Program, Duke-NUS Graduate Medical School, Singapore, Singapore; \\ e Department of Neurobiology, Caring Sciences and Society, Karolinska Institutet, \\ Stockholm, Sweden
}

\section{Key Words}

Cognitive impairment - Chinese older adults - Mini-Mental State Examination - Marital status . Social engagement

\section{Abstract}

Aims: To examine the association between marital status and cognitive impairment among community-dwelling Chinese older adults. Methods: We analyzed data from 2,498 Chinese aged 55 and older from the Singapore Longitudinal Aging Study cohort. Cognitive impairment was defined as a Mini-Mental State Examination total score of 23 or below. Odds ratios of associations were reported and adjusted for potential confounders in logistic regression models. Results: The prevalence of cognitive impairment was $12.2 \%(n=306)$. Being single was associated with about 2.5 times increased odds of cognitive impairment compared to being married (adjusted OR $=2.53,95 \% \mathrm{CI}$ : 1.41-4.55). The association between marital status and cognitive impairment was much stronger in men compared to that in women, and was indeed statistically significant only for men. Among the single and widowed persons social engagement was associated with a lower risk of cognitive impairment. Compared with subjects in the lowest tertile of social engagement scores, the odds of having cognitive impairment was lowered by $50 \%$ for subjects in the second and the third tertile. Conclusion: Being single or widowed was associated with higher odds of cognitive impairment compared to being married in a cohort of older Chinese men but not women. 


\section{Introduction}

The cognitive status in old age ranges from a cognitive function characteristic of normal adult functioning to severe cognitive impairment that is observed in dementia. Genetic, environmental, psychosocial and experiential factors across the entire life are important contributing factors.

The study of nongenetic factors is important because it is possible to develop evidencebased prevention programs to reduce the incidence of dementia if we can firmly establish the causality and relative contributions of multiple modifiable factors in the development of dementia [1,2]. To this end, previous research has provided evidence supporting the possible role of psychosocial factors (e.g., mentally stimulating activity, social engagement and physical exercise) $[3,4]$ as protective factors.

Few studies have examined the association between marital status and cognitive function in late life. Limited evidence from Caucasian populations suggests that being single increases one's risk of cognitive impairment. Data from the PAQUID (Personnes Agées QUID) cohort showed an increased risk of Alzheimer's disease among those who never married (relative risk $=2.68$ ) compared with married persons or cohabitants [5]. In the FINE (Finland, Italy and the Netherlands Elderly) study, men who had been living without a partner had at least a two times stronger cognitive decline later in life compared to men who had been living with a partner [6]. Another analysis based on data from the CAIDE (Cardiovascular Risk Factors, Aging and Dementia) study found that noncohabitants in midlife had about two times the risk of developing cognitive impairment later in life compared to cohabitants [7]. It is not known whether the same association exists among Asian elderly despite social, economic, cultural and lifestyle differences between the West and the East.

In the present study, we aimed to replicate and expand previous findings with data from a large sample of community-dwelling Chinese older adults. We hypothesized that: (1) being single or widowed would independently increase the risk of cognitive impairment in late life; (2) men would be more vulnerable to the effects of singlehood and widowhood than women, and (3) social engagement could protect single and widowed persons from cognitive impairment.

\section{Methods}

\section{Subjects}

The subjects in this study were 2,498 participants of the Singapore Longitudinal Aging Study (SLAS), a community-based epidemiologic study of aging and health. They were adults aged $\geq 55$ years living in the southeast region of Singapore. The methodology of the SLAS has been described in details elsewhere [8-12]. Briefly, a door-to-door census was conducted from September 2003 to November 2005, and eligible persons were invited to participate in the SLAS. A total of 2,808 adults participated in the SLAS with a major proportion of them being Chinese. The response rate was $78.2 \%$. Nonparticipants included those who were unable to complete the interview because they were too frail or terminally ill, such as those with stroke aphasia and profound dementia (8.5\%), or they refused or could not be contacted $(13.2 \%)$.

Due to the small proportion, the non-Chinese participants were omitted, giving us 2,607 Chinese subjects in the study. For this study, after excluding 106 participants with missing cognitive performance information and 3 participants with missing marital status information, we performed our analysis on the data of 2,498 participants.

The SLAS project was approved by the National University of Singapore Institutional Review Board. All participants signed an informed consent letter before participating. 
Feng et al.: Marital Status and Cognitive Impairment among Community-Dwelling Chinese Older Adults: The Role of Gender and Social Engagement

\section{Cognitive Performance}

The Mini-Mental State Examination (MMSE) test was used as a measure of global cognitive functioning [12-14]. Summed scores from the MMSE ranged from 0 to 30 where higher values denote better cognitive functioning. Cognitive impairment was defined as having MMSE scores of less than 24 points [14].

\section{Marital Status}

Information on the marital status was collected through a face-to-face questionnaire interview. Four categories were defined in the questionnaire for the SLAS: single, married, divorced/separated and widowed.

\section{Social and Productive Activities Score}

Social and productive activities were measured by the frequency $(0=$ never, $1=$ sometimes, $2=$ often) with which a subject engaged in six social activities (attending religious services, visiting cinemas/restaurants/sports events, day or excursion trips, playing cards/ games, joining senior citizen club activities and participating in social group activities such as karaoke and line dancing) and six productive activities (hobbies such as gardening/painting, preparing meals, shopping, paid community work, unpaid community work or other employment or business). The summed score of social activities (possible range of scores: $0-12$ ), the summed score of productive activities (possible range of scores: $0-12$ ) and the combined social and productive activities score (social engagement score, possible range of scores: 0-24) were analyzed as continuous variables. Social engagement scores in tertiles were used in the analysis of the association between social engagement and cognitive impairment among single and widowed persons.

\section{Other Data}

Related data included sociodemographic variables (age, gender and education), substance use (cigarette smoking and alcohol consumption), medical conditions, medications, blood pressure and fasting blood glucose. The 15-item Geriatric Depression Scale (GDS) [15], which was validated locally [16], was administered as a measure of depression. APOE genotyping was identified by PCR amplification followed by restriction endonuclease digestion of the PCR product. Physical activities were measured by the frequency $0=$ never, $1=$ sometimes, 2 = often) with which the respondents engaged in each of four fitness activities: physical exercise routines, walking, active sports or swimming and taiji. The summed physical activities score was used in the analysis as a continuous variable.

Hypertension was defined as having a systolic blood pressure of $>140 \mathrm{~mm} \mathrm{Hg}$ or a diastolic blood pressure of $>90 \mathrm{~mm} \mathrm{Hg}$ or a history of hypertension treatment. Diabetes mellitus was defined as a fasting blood glucose concentration of $\geq 7.0 \mathrm{mmol} / \mathrm{l}$ or a history of treatment for diabetes mellitus. Depression was defined as a GDS total score of $\geq 5$.

\section{Statistical Analysis}

We first computed frequencies and other descriptive statistics to find out the characteristics of the sample. The associations between variables and marital status were assessed by $\chi^{2}$ tests for the categorical variables and by analyses of variance for the continuous variables.

Hierarchical logistic regression was used to examine the relationship between marital status and cognitive impairment. Odds ratios (ORs) of having cognitive impairment with $95 \%$ confidence intervals (CIs) were obtained from the fitted models where we specified the married group as the control category. The base model (model 1) consists of the marital status only. In model 2, we adjusted additionally for age, gender and education; smoking and alcohol consumption, hypertension, diabetes mellitus, heart diseases, stroke, physical activity, 
Feng et al.: Marital Status and Cognitive Impairment among Community-Dwelling Chinese Older Adults: The Role of Gender and Social Engagement

Table 1. Characteristics of study subjects by marital status

\begin{tabular}{lccccc}
\hline Characteristic & Marital status & & & $p$ \\
\cline { 2 - 5 } & $\begin{array}{l}\text { married } \\
(\mathrm{n}=1,857)\end{array}$ & $\begin{array}{l}\text { single } \\
(\mathrm{n}=151)\end{array}$ & $\begin{array}{l}\text { divorced, } \\
\text { separated }(\mathrm{n}=76)\end{array}$ & $\begin{array}{c}\text { widowed } \\
(\mathrm{n}=414)\end{array}$ \\
\hline Age, years & $64.89 \pm 6.99$ & $65.13 \pm 7.89$ & $64.11 \pm 6.54$ & $71.47 \pm 8.52$ & $<0.0001$ \\
Women & $1,052(56.6)$ & $107(70.9)$ & $59(77.6)$ & $376(90.8)$ & $<0.0001$ \\
Education, primary and below & $908(48.9)$ & $68(45.0)$ & $42(55.3)$ & $307(74.2)$ & $<0.0001$ \\
Current and ex cigarette smoker & $328(17.7)$ & $28(18.5)$ & $11(14.5)$ & $42(10.1)$ & 0.002 \\
Regular alcohol drinker & $225(12.1)$ & $6(4.0)$ & $10(13.2)$ & $21(5.1)$ & $<0.0001$ \\
Physical activities score & $2.65 \pm 2.03$ & $2.39 \pm 1.77$ & $1.97 \pm 2.05$ & $2.20 \pm 1.98$ & $<0.0001$ \\
Social activities score & $3.22 \pm 2.53$ & $2.82 \pm 2.51$ & $3.51 \pm 2.69$ & $3.09 \pm 2.35$ & 0.149 \\
Productive activities score & $3.80 \pm 1.86$ & $3.68 \pm 2.39$ & $4.11 \pm 1.70$ & $3.90 \pm 1.86$ & 0.323 \\
Hypertension & $987(53.1)$ & $63(41.7)$ & $46(60.5)$ & $269(65.0)$ & $<0.0001$ \\
Diabetes & $289(15.6)$ & $21(13.9)$ & $10(13.2)$ & $86(20.8)$ & 0.046 \\
Heart disease & $106(5.7)$ & $12(7.9)$ & $3(4.0)$ & $22(5.3)$ & 0.591 \\
Stroke & $70(3.8)$ & $5(3.3)$ & $5(6.6)$ & $13(3.1)$ & 0.517 \\
APOE $\varepsilon 4$ carrier & $297(16.0)$ & $20(13.3)$ & $21(27.6)$ & $64(15.5)$ & 0.043 \\
Depression (GDS score $\geq 5)$ & $227(12.2)$ & $33(21.8)$ & $18(23.7)$ & $63(15.2)$ & $<0.0001$ \\
MMSE total score & $27.31 \pm 3.01$ & $25.80 \pm 5.86$ & $27.66 \pm 2.68$ & $25.31 \pm 4.46$ & $<0.0001$ \\
\hline
\end{tabular}

Figures are means \pm SD or numbers with percentages in parentheses.

APOE $\varepsilon 4$ carrier status and depression were further adjusted in model 3 , and social and productive activities were further adjusted in model 4.

Multiple logistic regressions based only on single and widowed subjects were performed to examine the association between social engagement (combination of social and productive activities) and cognitive impairment. The relationship between social engagement scores and MMSE total scores was shown using a scatter plot. All data analyses were performed by SAS 9.1 (SAS Institute Inc., Cary, N.C., USA).

\section{Results}

Among these 2,498 subjects, about $74.3 \%$ of them were married, $6.1 \%$ were single, $3.0 \%$ divorced or separated and $16.6 \%$ were widowed. The prevalence of cognitive impairment (defined as an MMSE score $\leq 23$ ) was $12.2 \%(\mathrm{n}=306)$. As shown in table 1 , subjects who were widowed were generally older. This group had the highest percentage of women and subjects who had primary education and below. They also had the highest occurrence of hypertension and diabetes mellitus. Singles had the highest proportion of smokers but the lowest proportion of alcohol drinkers and the lowest mean score for social and productive activities. We also observed a high frequency of heart disease in the group of singles compared to the other two categories. Married persons had the highest mean score for physical activities and the lowest occurrence of depression while the divorced or separated had the highest occurrence of stroke and the highest percentage of APOE $\varepsilon 4$ carriers. The widowed persons had the lowest mean MMSE score, as compared to the other three groups. Various characteristics of male and female subjects based on marital status are shown in table 2.

In the logistic regression analysis results shown in table 3, compared to married participants, single persons had about 2.5 times increased odds of cognitive impairment. Moreover, 
Feng et al.: Marital Status and Cognitive Impairment among Community-Dwelling Chinese Older Adults: The Role of Gender and Social Engagement

Table 2. Characteristics of study subjects by gender and marital status

\begin{tabular}{|c|c|c|c|c|c|}
\hline \multirow[t]{2}{*}{ Characteristic } & \multicolumn{4}{|l|}{ Marital status } & \multirow[t]{2}{*}{$\mathrm{p}$} \\
\hline & married & single & divorced, separated & widowed & \\
\hline Men & $(n=805)$ & $(n=44)$ & $(n=17)$ & $(n=38)$ & \\
\hline Age, years & $66.26 \pm 7.49$ & $70.23 \pm 7.43$ & $63.08 \pm 5.96$ & $78.31 \pm 78.90$ & $<0.0001$ \\
\hline Education, primary and below & $314(39.0)$ & $32(72.7)$ & $8(47.1)$ & $23(60.5)$ & $<0.0001$ \\
\hline Current and ex cigarette smoker & $290(36.0)$ & $22(50.0)$ & $8(47.1)$ & $12(31.6)$ & 0.197 \\
\hline Regular alcohol drinker & $184(22.9)$ & $3(6.8)$ & $5(29.1)$ & $4(10.5)$ & 0.020 \\
\hline Physical activities score & $2.87 \pm 2.02$ & $2.25 \pm 1.66$ & $2.12 \pm 1.97$ & $1.41 \pm 1.91$ & $<0.0001$ \\
\hline Social activities score & $2.73 \pm 2.29$ & $1.57 \pm 2.13$ & $2.00 \pm 1.71$ & $1.97 \pm 1.86$ & 0.001 \\
\hline Productive activities score & $3.09 \pm 1.90$ & $1.93 \pm 1.89$ & $3.29 \pm 1.80$ & $2.89 \pm 2.22$ & 0.001 \\
\hline Hypertension & $458(56.9)$ & $26(59.1)$ & $10(58.8)$ & $26(68.4)$ & 0.566 \\
\hline Diabetes & $147(18.3)$ & 7 (15.9) & $3(17.6)$ & $5(13.2)$ & 0.856 \\
\hline Heart disease & $73(9.1)$ & $6(13.6)$ & $3(17.6)$ & $5(13.2)$ & 0.396 \\
\hline Stroke & $44(5.5)$ & $5(11.4)$ & $3(17.6)$ & $0(0)$ & 0.025 \\
\hline APOE $\varepsilon 4$ carrier & $126(15.7)$ & $4(9.1)$ & $7(41.2)$ & $6(15.8)$ & 0.047 \\
\hline Depression (GDS score $\geq 5$ ) & $94(11.7)$ & $15(34.1)$ & $4(23.5)$ & $11(28.9)$ & $<0.0001$ \\
\hline MMSE total score & $27.76 \pm 2.54$ & $22.89 \pm 6.94$ & $27.59 \pm 2.45$ & $25.61 \pm 4.21$ & $<0.0001$ \\
\hline Women & $(n=1,052)$ & $(\mathrm{n}=107)$ & $(n=59)$ & $(n=376)$ & \\
\hline Age, years & $63.84 \pm 6.39$ & $63.04 \pm 7.10$ & $64.40 \pm 6.72$ & $70.78 \pm 8.18$ & $<0.0001$ \\
\hline Education, primary and below & $594(56.5)$ & $36(33.6)$ & $34(57.6)$ & $284(75.5)$ & $<0.0001$ \\
\hline Current and ex cigarette smoker & $38(3.6)$ & $6(5.6)$ & $3(5.1)$ & $30(8.0)$ & 0.009 \\
\hline Regular alcohol drinker & $41(3.9)$ & $3(2.8)$ & $5(8.5)$ & $17(4.5)$ & 0.313 \\
\hline Physical activities score & $2.47 \pm 2.01$ & $2.44 \pm 1.82$ & $1.93 \pm 2.10$ & $2.28 \pm 1.98$ & 0.121 \\
\hline Social activities score & $3.60 \pm 2.64$ & $3.36 \pm 2.49$ & $3.95 \pm 2.77$ & $3.20 \pm 2.37$ & 0.037 \\
\hline Productive activities score & $4.35 \pm 1.63$ & $4.43 \pm 2.19$ & $4.35 \pm 1.61$ & $4.00 \pm 1.80$ & 0.007 \\
\hline Hypertension & $529(50.3)$ & $37(34.6)$ & $36(61.0)$ & $243(64.6)$ & $<0.0001$ \\
\hline Diabetes & $142(13.6)$ & $14(13.1)$ & 7 (11.9) & $81(21.5)$ & 0.002 \\
\hline Heart disease & $33(3.1)$ & $6(5.6)$ & $0(0)$ & $17(4.5)$ & 0.170 \\
\hline Stroke & $26(2.5)$ & $0(0)$ & $2(3.4)$ & $13(3.5)$ & 0.240 \\
\hline APOE $\varepsilon 4$ carrier & $171(16.3)$ & $16(15.0)$ & $14(23.7)$ & $58(15.4)$ & 0.386 \\
\hline Depression (GDS score $\geq 5$ ) & $133(12.6)$ & $18(16.8)$ & $14(23.7)$ & $52(13.8)$ & 0.076 \\
\hline MMSE total score & $26.97 \pm 3.28$ & $27.00 \pm 4.91$ & $27.68 \pm 2.76$ & $25.28 \pm 4.49$ & $<0.0001$ \\
\hline
\end{tabular}

Figures are means \pm SD or numbers with percentages in parentheses.

after adjusting for possible confounders, the widowed group did not show a significant difference in odds of cognitive impairment compared to the married participants. No significant associations were found with cognitive impairment for being either divorced or widowed. Stratifying by gender showed significant results among the men. Single men had about a 6.2 times and widowed men had about a 5 times increased risk of cognitive impairment at the time of cognitive measurement compared to the married men. Women living without a partner, however, did not have a significantly higher risk of cognitive impairment at baseline compared to the married women. The interaction term marital status $\times$ gender was statistically significant in the whole study sample ( $p=0.0209$ in the fully adjusted model 4$)$.

Table 4 shows that, among the single and widowed persons, an increase in social engagement was associated with a lower risk of cognitive impairment. Compared with subjects in the lowest tertile of social engagement scores, the adjusted OR of cognitive impairment was 0.50 for subjects in the second (95\% CI: 0.27-0.92) and the third tertile (95\% CI: $0.25-1.01)$. The scatter plot showed a positive correlation between social engagement scores and the MMSE total scores (Pearson correlation: 0.417, p < 0.001) (fig. 1). 
Feng et al.: Marital Status and Cognitive Impairment among Community-Dwelling Chinese Older Adults: The Role of Gender and Social Engagement

Table 3. Association between marital status and cognitive impairment

\begin{tabular}{lclll}
\hline Marital status & Model 1 & Model 2 & Model 3 & Model 4 \\
\hline Men & & & & \\
$\quad$ Married & 1.00 (reference) & 1.00 (reference) & 1.00 (reference) & 1.00 (reference) \\
$\quad$ Single & $11.44(5.78-22.62)$ & $7.26(3.41-15.46)$ & $6.58(2.71-15.97)$ & $6.23(2.46-15.78)$ \\
$\quad$ Divorced or separated & $1.14(0.15-8.77)$ & $1.90(0.23-15.85)$ & $0.92(0.09-9.85)$ & $0.97(0.09-10.75)$ \\
$\quad$ Widowed & $7.40(3.44-15.93)$ & $2.35(0.93-5.95)$ & $4.02(1.34-12.06)$ & $4.96(1.55-15.86)$ \\
Women & & & & \\
$\quad$ Married & $1.00($ reference) & 1.00 (reference) & 1.00 (reference) & 1.00 (reference) \\
$\quad$ Single & $0.99(0.54-1.82)$ & $1.55(0.74-3.23)$ & $1.74(0.82-3.72)$ & $1.53(0.67-3.45)$ \\
$\quad$ Divorced or separated & $0.52(0.19-1.46)$ & $0.45(0.15-1.33)$ & $0.50(0.16-1.56)$ & $0.54(0.17-1.76)$ \\
$\quad$ Widowed & $2.22(1.64-3.00)$ & $0.88(0.61-1.26)$ & $0.92(0.62-1.36)$ & $0.98(0.66-1.47)$ \\
Total & & & \\
$\quad$ Married & $1.00($ reference) & $1.00($ reference) & $1.00($ reference) & $1.00($ reference) \\
$\quad$ Single & $2.44(1.59-3.76)$ & $2.94(1.77-4.89)$ & $2.82(1.62-4.90)$ & $2.53(1.41-4.55)$ \\
$\quad$ Divorced or separated & $0.69(0.28-1.74)$ & $0.59(0.22-1.56)$ & $0.59(0.21-1.66)$ & $0.61(0.21-1.79)$ \\
$\quad$ Widowed & $3.14(2.39-4.13)$ & $1.04(0.74-1.45)$ & $1.14(0.79-1.63)$ & $1.22(0.84-1.77)$ \\
\hline
\end{tabular}

Figures are ORs with 95\% CIs in parentheses. Model 1 = Base model; model 2 = model $1+$ age, gender and education; model 3 = model $2+$ smoking and alcohol consumption, hypertension, diabetes mellitus, heart diseases, stroke, physical activity, APOE $\varepsilon 4$ carrier status and depression; model $4=$ model $3+$ social and productive activities.

Table 4. Association between social engagement and cognitive impairment in single and widowed persons $(\mathrm{n}=560)$

\begin{tabular}{llll}
\hline \multicolumn{3}{l}{ Social engagement score } & \\
\cline { 2 - 4 } & $\begin{array}{l}\text { first tertile }(\mathrm{n}=198) \\
\text { (reference) }\end{array}$ & second tertile $(\mathrm{n}=198)$ & third tertile $(\mathrm{n}=164)$ \\
\hline Model 1 & 1 & $0.28(0.17-0.45)$ & $0.23(0.13-0.39)$ \\
Model 2 & 1 & $0.40(0.23-0.69)$ & $0.48(0.25-0.89)$ \\
Model 3 & 1 & $0.40(0.23-0.71)$ & $0.42(0.21-0.82)$ \\
Model 4 & 1 & $0.50(0.27-0.92)$ & $0.50(0.25-1.01)$
\end{tabular}

Figures are ORs with 95\% CIs in parentheses. First tertile $=0-5$; second tertile $=6-8$; third tertile $=\geq 9$; model 1 = base model; model $2=$ model $1+$ age, gender and education; model $3=$ model $2+$ smoking and alcohol consumption, hypertension, diabetes mellitus, heart diseases, stroke, physical activity, APOE $\varepsilon 4$ carrier status and depression; model $4=$ model $3+$ social and productive activities.

\section{Discussion}

Based on data from a large group of older Chinese adults in the SLAS cohort, we observed that singlehood and widowhood increased the odds of cognitive impairment independent of a wide range of known factors that contribute to cognitive function in late life, including social engagement. The association was much stronger in men than in women and was only statistically significantly in men. Social engagement was associated with reduced odds of having cognitive impairment among single and widowed persons.

Our findings are consistent with the results from a handful of previous studies that had been conducted in Caucasian populations $[5-7,17]$ and is in fact the first report on the asso- 


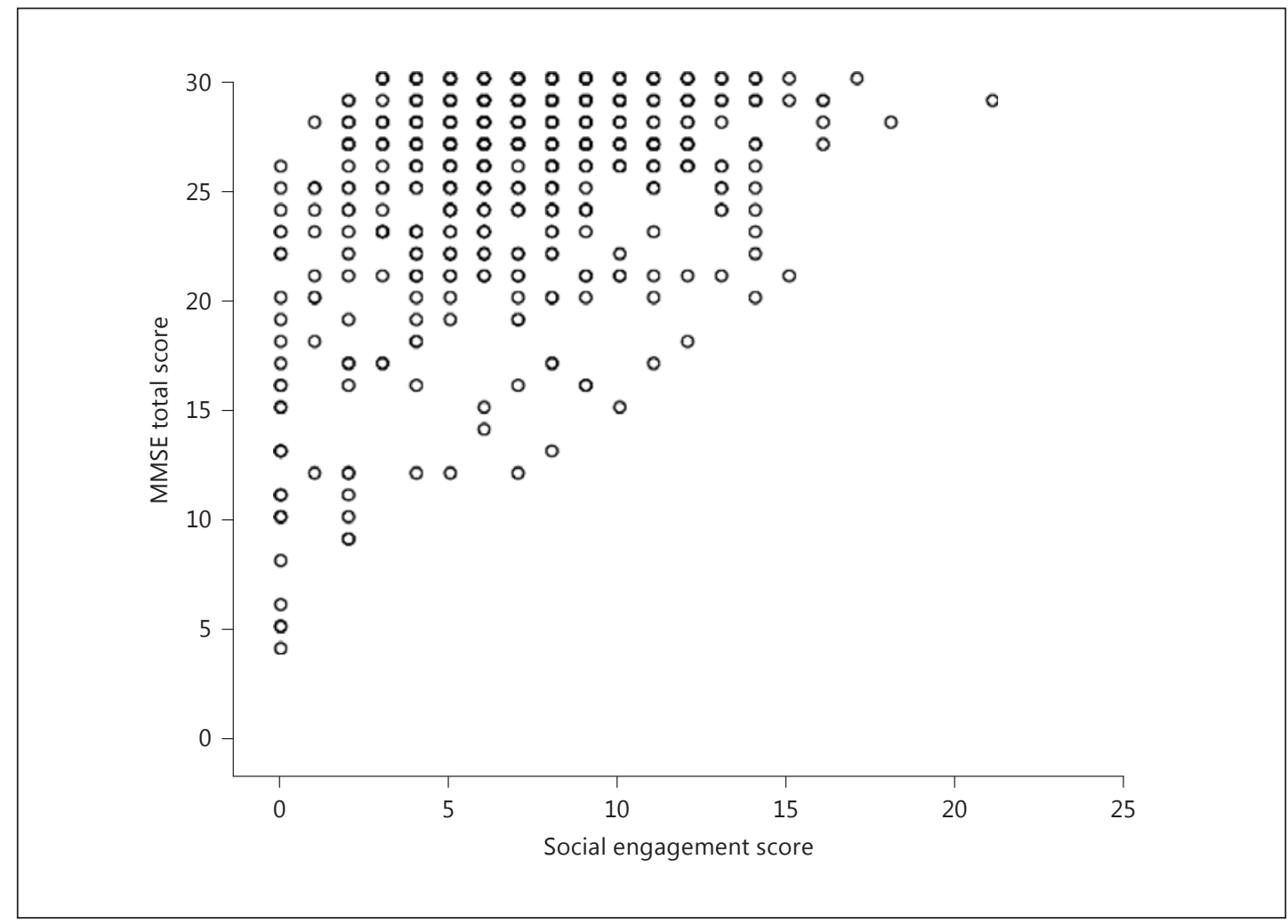

Fig. 1. The association between social engagement scores and MMSE total scores in single or widowed persons $(\mathrm{n}=560)$.

ciation between marital status and cognitive status in late life from an Asian population. Our findings suggest that a lasting relationship such as marriage has protective benefits on cognitive function. Although our data were collected cross-sectionally, it is in fact a retrospective cohort study in nature as marital status exposure happened before the study participants' cognitive functions were assessed. We assume that information bias on a simple fact such as marital status would be minimal, if not negligible. With the above arguments, a longitudinal relationship between marital status and cognitive impairment is supported by our results.

We propose that the observed association between marital status and cognitive impairment can be largely explained by psychological benefits that are associated with being in a relationship. Married individuals may be exposed to more cognitive and social engagement than those who are not married, and the cumulative effects of such engagements can protect them from cognitive impairment in late life. A married individual may also experience less psychological stress and feelings of loneliness because of the available emotional support and connectedness from a close companion. Given the important roles that a married man or woman need to play, married persons may also have a greater purpose in life. Those psychological benefits can provide additional protection in addition to cognitively engaging activities, as previous studies have shown that each of these factors, namely, psychological stress [18], loneliness [19] or greater purpose in life [20] was associated with increased or reduced risk of dementia.

We observed that singles had elevated odds of cognitive impairment but the association was not statistically significant for those who were widowed or divorced. In men, there was 
Feng et al.: Marital Status and Cognitive Impairment among Community-Dwelling

a stronger association for singlehood $(\mathrm{OR}=6.23)$ than widowhood $(\mathrm{OR}=4.96)$. A possible explanation for the observations could be different time exposures of living without a partner. Since our data were collected with a cross-sectional design in late life, a person categorized as 'single' would have never been married while a person categorized as widowed or divorced could have entered into that category late in life. In line with this hypothesis, in the study of Håkansson et al. [7], the highest ORs were observed for those who were widowed/divorced in midlife and still so on average 21 years later.

It is known that social engagement, which is defined by the maintenance of many social connections and a high level of participation in social activities, is a protective factor for cognitive impairment $[2,17,21]$. In our multiple logistic regression model, the summed social and productive activities scores were controlled for as a measure of social engagement, and the association between marital status and cognitive impairment persisted in the final model. However, the summed scores here reflect a person's participation in activities and do not measure the quantity and quality of one's social network; hence, we cannot rule out the possibility that social isolation may still play a major role in mediating the association between singlehood/widowhood and cognitive impairment. Notably, social engagement was associated with lowered odds of having cognitive impairment among those who were single or widowed, which supports the importance of its protective role in preserving cognitive function.

We found that the association between the marital status and cognitive impairment was significant in men but not women. The findings differ from those in the West as recent research supports that the average mental health benefits associated with entering marriage are similar for men and women [22]. In the study of Håkansson et al. [7], the increase in risk of dementia was significant for both men and women, with slightly higher ORs for men. Although there is no simple explanation for the observed gender difference in the cognitive benefits of marriage in our study sample of Chinese older adults, gender differences in patterns of friendships and social networks may provide an explanation. Women, in general, have more intimate and personal friendships and social networks than men [23, 24], which can compensate for the lack of intimacy and companionship in singlehood. In our study sample, the levels of participation in social activities and productive activities were much higher in women than in men (table 2).

The study has several limitations: (1) a cause-effect relationship between marital status and cognitive impairment cannot be established with the cross-sectional design of data collection; (2) residual confounding from unmeasured variables was possible and may have contributed to the observed associations, and (3) with a nonresponse rate of $21.8 \%$, the influence of a participation bias could not be ruled out. Also, the study findings were based on a group of community-dwelling Asian older adults in Singapore and may not be generalizable to other populations.

\section{Conclusion}

In conclusion, we found that marriage was associated with reduced odds of having cognitive impairment among community-dwelling Chinese men but not women. This finding should be interpreted with caution and must be replicated and further examined with more studies of other populations. Since social engagement was associated with lowered cognitive impairment among those who are single or widowed, psychosocial interventions could be offered to this high-risk subgroup to reduce the incidence of dementia. 
Feng et al.: Marital Status and Cognitive Impairment among Community-Dwelling Chinese Older Adults: The Role of Gender and Social Engagement

\section{Acknowledgements}

TheSLAS was supported by research grants No.03/1/21/17/214 and No. 08/1/21/19/567 from the Biomedical Research Council, Agency for Science, Technology and Research in Singapore.

\section{Disclosure Statement}

The authors declare that they have no financial support or relationships that may pose conflicts of interest. No commercial company sponsored or played any role in the design and methods of the study, subject recruitment, data collection and analysis, and preparation of this article.

\section{References}

1 Ritchie K, Carrière I, Ritchie CW, Berr C, Artero S, Ancelin M-L: Designing prevention programmes to reduce incidence of dementia: prospective cohort study of modifiable risk factors. BMJ 2010;341:c3885.

2 Feng L, Chiu H, Chong M-Y, Yu X, Kua E-H: Dementia in Chinese populations: current data and future research. Asia Pac Psychiatry 2011;3:109-114.

-3 Qiu C, Xu W, Fratiglioni L: Vascular and psychosocial factors in Alzheimer's disease: epidemiological evidence toward intervention. J Alzheimers Dis 2010;20:689-697.

-4 Verghese J, Lipton RB, Katz MJ, Hall CB, Derby CA, Kuslansky G, Ambrose AF, Sliwinski M, Buschke H: Leisure activities and the risk of dementia in the elderly. N Engl J Med 2003;348:2508-2516.

5 Helmer C, Damon D, Letenneur L, Fabrigoule C, Barberger-Gateau P, Lafont S, Fuhrer R, Antonucci T, Commenges D, Orgogozo JM, Dartigues JF: Marital status and risk of Alzheimer's disease - a French population-based cohort study. Neurology 1999;53:1953-1958.

6 van Gelder BM, Tijhuis M, Kalmijn S, Giampaoli S, Nissinen A, Kromhout D: Marital status and living situation during a 5-year period are associated with a subsequent 10 -year cognitive decline in older men: the FINE study. J Gerontol B Psychol Sci Soc Sci 2006;61:P213-P219.

7 Håkansson K, Rovio S, Helkala EL, Vilska AR, Winblad B, Soininen H, Nissinen A, Mohammed AH, Kivipelto M: Association between mid-life marital status and cognitive function in later life: population based cohort study. BMJ 2009;339:b2462.

8 Ng TP, Feng L, Niti M, Kua EH, Yap KB: Tea consumption and cognitive impairment and decline in older Chinese adults. Am J Clin Nutr 2008;88:224-231.

-9 Feng L, Ng TP, Chuah L, Niti M, Kua EH: Homocysteine, folate, and vitamin B-12 and cognitive performance in older Chinese adults: findings from the Singapore longitudinal ageing study. Am J Clin Nutr 2006;84:1506-1512.

10 Feng L, Li J, Yap KB, Kua EH, Ng TP: Vitamin B-12, apolipoprotein E genotype, and cognitive performance in community-living older adults: evidence of a gene-micronutrient interaction. Am J Clin Nutr 2009;89:12631268.

11 Feng L, Gwee X, Kua EH, Ng TP: Cognitive function and tea consumption in community dwelling older Chinese in Singapore. J Nutr Health Aging 2010;14:433-438.

$\$ 12$ Feng L, Chong MS, Lim WS, Ng TP: The Modified Mini-Mental State Examination test: normative data for Singapore Chinese older adults and its performance in detecting early cognitive impairment. Singapore Med J 2012;53:458-462.

13 Folstein MF, Folstein SE, McHugh PR: 'Mini-mental state'. A practical method for grading the cognitive state of patients for the clinician. J Psychiatr Res 1975;12:189-198.

14 Ng T-P, Niti M, Chiam P-C, Kua E-H: Ethnic and educational differences in cognitive test performance on MiniMental State Examination in Asians. Am J Geriatr Psychiatry 2007;15:130-139.

15 Sheikh JI, Yesavage JA: Geriatric Depression Scale (GDS): recent evidence and development of a shorter version; in Brink TL (ed): Clinical Gerontology: A Guide to Assessment and Intervention. New York, The Haworth Press, 1986, pp 165-173.

16 Lim PP, Ng LL, Chiam PC, Ong PS, Ngui FT, Sahadevan S: Validation and comparison of three brief depression scales in an elderly Chinese population. Int J Geriatr Psychiatry 2000;15:824-830.

17 Fratiglioni L, Wang H-X, Ericsson K, Maytan M, Winblad B: Influence of social network on occurrence of dementia: a community-based longitudinal study. Lancet 2000;355:1315-1319.

18 Johansson L, Guo X, Waern M, Östling S, Gustafson D, Bengtsson C, Skoog I: Midlife psychological stress and risk of dementia: a 35-year longitudinal population study. Brain 2010;133:2217-2224. 
19 Wilson RS, Barnes LL, Aggarwal NT, Boyle PA, Hebert LE, Mendes de Leon CF, Evans DA: Cognitive activity and the cognitive morbidity of Alzheimer disease. Neurology 2010;75:990-996.

20 Boyle PA, Buchman AS, Barnes LL, Bennett DA: Effect of a purpose in life on risk of incident Alzheimer disease and mild cognitive impairment in community-dwelling older persons. Arch Gen Psychiatry 2010;67:304310.

-21 Bassuk SS, Glass TA, Berkman LF: Social disengagement and incident cognitive decline in community-dwelling elderly persons. Ann Intern Med 1999;131:165-173.

22 Williams K, Frech A, Calson DL: Marital status and mental health; in Scheid TL, Brown TN (eds): A Handbook for the Study of Mental Health: Social Contexts, Theories, and Systems. Cambridge, Cambridge University Press, 2009, pp 306-320.

23 Lee GR, DeMaris A, Bavin S, Sullivan R: Gender differences in the depressive effect of widowhood in later life. J Gerontol B Psychol Sci Soc Sci 2001;56:S56-S61.

24 Elkins L, Peterson C: Gender differences in best friendships. Sex Roles 1993;29:497-508. 